\title{
Correlation between microRNA-34a levels and lens opacity severity in age-related cataracts
}

K-H Chien ${ }^{1}$, S-J Chen ${ }^{2,3}$, J-H Liu'², ${ }^{2}, \mathrm{H}-\mathrm{M}$ Chang $^{5}$, L-C Woung ${ }^{2,6}$, C-M Liang ${ }^{1}$, J-T Chen', T-J Lin',8, S-H Chiou ${ }^{2,3,9,10}$ and C-H Peng ${ }^{2,9,11}$

\section{Abstract}

Purpose MicroRNA 34a (miR-34a) is involved in regulating tissue senescence. However, the role of miR-34a in age-related cataracts is unclear. In this study, we evaluated the correlations among the severity of lens opacity, patient age, and miR-34a expression level in the lens epithelium of age-related cataracts for clarifying the role of miR-34a in the lens senescence. Methods This study was carried as a case control study in the Department of Ophthalmology, Taipei Veterans General Hospital, Taiwan. We recorded age of each patient at the time of their cataract surgery and information regarding lens opacity according to a modified version of the Lens Opacities Classification System III.

Correlations among age, lens opacity, and miR-34a expression levels were evaluated. Results This study evaluated 110 patients with a mean age of 73.19 years $(S D \pm \mathbf{1 0 . 2})$. Older patients had higher nuclear cataract (NC), cortical (C), and posterior subcapsular cataract $(\mathrm{P})$ scores (one-way analysis of variance (ANOVA), $P<0.05$ ). miR-34a expression levels were significantly different between each age group (ANOVA post hoc Bonferroni's test, $P<0.001$ ), and there were moderate correlations between high $\mathrm{NC}, \mathrm{C}$, and $P$ cataract scores and high miR-34a levels (Pearson correlation coefficient; $R=0.606,0.575$, and 0.515 , respectively).

Conclusions The current study demonstrated positive correlations between high miR-34a levels and high lens opacity severity in NC, C, or $\mathrm{P}$ cataracts. These results suggest that miR34a expression has a role in lens senescence. Eye (2013) 27, 883-888; doi:10.1038/eye.2013.90; published online 10 May 2013

Keywords: aging; cataract; microRNA; senescence

\section{Introduction}

Studies have provided many evidences of the participation of microRNAs in multiple cellular functions, such as cell proliferation, ${ }^{1}$ cell apoptosis, ${ }^{2}$ stress response, ${ }^{3}$ and senescence, ${ }^{4}$ mainly through post-transcriptional mRNA silencing and subsequent protein translation inhibition. MicroRNAs, which are groups of endogenous and small noncoding RNAs, have also been found to be biological modulators at the post-translational level through complementary or partial complementary binding with the $3^{\prime}$ untranslated region (UTR) of target mRNA. ${ }^{5,6}$ MicroRNAs are also involved in DNA damage response-induced senescence, which is mainly governed by the p53 pathway. $^{7}$ The microRNA-34a (miR-34a), the most apparent of the p53-induced microRNAs, has a role in senescence through the upregulation of acetylated p53 and activation of apoptosis in a positive feedback loop. ${ }^{2}$ However, very less information is available regarding miR-34a in ocular aging processes, such as cataracts.

Cataracts, the most common cause of blindness worldwide, ${ }^{8}$ are significantly related to the aging process. Currently, many factors such as diabetes mellitus, ultraviolet exposure, systemic drugs, and other ocular diseases are known to be related to cataract formation. Among these disorders, oxidative stress with the subsequent formation of reactive oxygen species reactive oxygen species (ROS) is thought to be a major predisposing factor in age-related cataracts. ${ }^{9}$ Recently, a biological factor, silent information regulator T1 (SirT1), was proposed as an element involved in delaying the aging process and lifespan extension by suppressing $\mathrm{NF}-\kappa \mathrm{B}$ signaling. We have found that decreased expression of the SirT1 gene is associated with higher cataract severity and patient age in our prior study, ${ }^{10}$ but miR-34a expression in age-related cataracts has not been understood well.
'Department of Ophthalmology, Tri-Service General Hospital, National Defense Medical Center Taipei, Taiwan

${ }^{2}$ School of Medicine, National Yang-Ming University, Taipei, Taiwan

${ }^{3}$ Department of Ophthalmology, Taipei Veterans General Hospital, Taipei, Taiwan

${ }^{4}$ Department of Ophthalmology, Cheng-Hsin General Hospital, Taipei, Taiwan

${ }^{5}$ Department of Optics and Photonics, National Central University, Chung-Li, Taiwan

${ }^{6}$ Department of Ophthalmology, Taipei City Hospital, Taipei, Taiwan

The Graduate Institute of Clinical Medical Sciences, Chang Gung University College of Medicine; Kaohsiung, Kaohsiung, Taiwan

${ }^{8}$ Department of Ophthalmology، Zuoying Armed Forces General Hospital, Kaohsiung, Taiwan

Institute of Clinical Medicine, National Yang-Ming University, Taipei, Taiwan

${ }^{10}$ Department of Medical Research and Education, Taipei Veterans General Hospital, Taipei, Taiwan

${ }^{1}$ Department of Ophthalmology, Shin-Kong Wu Ho-Su Memorial Hospital and Fu-Jen Catholic University, Taipei, Taiwan

Correspondence:

C-H Peng, Department of Ophthalmology, Shin Kong Wu Ho-Su Memorial Hospital and Fu-Jen Catholic University, No.95, Wenchang Rd., Shilin District, Taipei 111, Taiwan.

Tel: +886 228332211 .

Fax: +886 228331111

E-mail: chpeng1008@gmail.com

Received: 28 June 2012 Accepted: 20 March 2013 Published online: 10 May 2013

This paper is not presented at any conference. 
The aim of this study was to investigate the expression of miR-34a in the lens epithelium of patients with age-related cataracts. To further evaluate the correlations among patient age, cataract severity, and miR-34a expression, the Lens Opacities Classification System III (LOC III) ${ }^{11}$ was conducted to stratify the patients according to cataract type and severity.

\section{Materials and methods}

Lens epithelium samples were obtained from 110 eyes between January 2008 and December 2010. This study followed the tenets of the Declaration of Helsinki. All patients underwent a complete preoperative ophthalmologic examination and only those who had no ocular diseases other than age-related cataracts were included. Patients who had previously undergone ocular surgery or those with diabetes were not included from this study. All samples were collected after obtaining informed consent from the patient. Lens epithelium samples were obtained by intact continuous curvilinear capsulorhexis, with care taken to avoid vascular contact or damage to the iris or other intraocular structures.

Cataract type and severity were graded and recorded on the basis of a modified version of the LOCSIII by using six silt-lamp images for grading nuclear color and nuclear retro-illumination images for grading posterior subcapsular $(\mathrm{P})$ cataracts. Each scale on the LOCSIII is a decimalized scale ranging from 0.1 (a completely clear or colorless lens) to 5.9 (upper value on the $\mathrm{C}$ and $\mathrm{P}$ scales indicating complete opacification of the cortex or posterior capsule) and 6.9 on the NC scales (indicating advanced opacification and brunescence of the nucleus). All LOCSIII scorings among subjects was carried out and consisted up to at least three ophthalmologists. The control lens epithelium samples with a LOCSIII score of C1, NC1, and P1 were obtained from the vitrectomy operation for epiretinal membranes $(N=13)$.

\section{MicroRNA isolation and microRNA quantitative real-time reverse transcriptase (RT-PCR)}

The expression levels of miR-34a microRNA were determined by quantitative real-time RT-PCR (qRT-PCR) as previously described. ${ }^{12}$ Briefly, a mirVana PARIS kit (Ambion, Grand Island, NY, USA) was used to isolate small RNAs from total RNA according to the manufacturer's instructions. The mature miR-34a sequence is $\left(5^{\prime}-3^{\prime}\right)$ UGGCAGUGUCUUAGCUGGUUGUU. For microRNA quantitation, qRT-PCR was performed using TaqMan miRNA assays (Applied Biosystems, Grand Island, NY, USA) with specific primer sets. All reagents and protocols were obtained from (Applied Biosystems) and detection was performed using a 7900HT fast realtime PCR system (Applied Biosystems) with RNU6B as an internal control. The conditions for amplification were as follows: one cycle of $94^{\circ} \mathrm{C}$ for $2 \mathrm{~min}$, followed by 50 cycles of $94{ }^{\circ} \mathrm{C}$ for $20 \mathrm{~s}, 57^{\circ} \mathrm{C}$ for $20 \mathrm{~s}$, and $70{ }^{\circ} \mathrm{C}$ for $20 \mathrm{~s}$.

MicroRNA-specific qRT-PCR was performed in triplicate and repeated three times, the expression ratio of miR-34a/ RUN6B in control patients was defined as 1 .

\section{Statistical methods}

For statistical analysis, patient age was expressed as mean \pm SD and miR-34a mRNA levels were expressed as mean \pm SE. The correlations among LOCSIII score, age, and levels of miR-34a mRNA were analyzed using oneway analysis of variance (ANOVA), followed by post hoc Bonferroni's test. The correlation between LOCSIII score and age, and LOCSIII score and levels of miR-34a mRNA expression were analyzed using the Pearson correlation coefficient and unpaired Student's $t$-test. A $P$-value of $<0.05$ was considered statistically significant. All analyses were performed using SPSS 12.0 (IBM, Armonk, NY, USA).

\section{Results}

At the end of enrollment, 110 patients were included in this study. Patient ages ranged from 55-92 years with an average of 73.19 years (SD 10.2). We further stratified patients according to age into four groups: between 55 and 64 years $(25 ; 22.7 \%)$, between 65 and 74 years (35; $31.8 \%)$, between 75 and 84 years $(28 ; 25.5 \%)$, and older than 85 years $(22 ; 20 \%)$.

Considering the entire patient cohort, the age at the time of cataract surgery showed significant differences with respect to the grading of nuclear cataracts (NCs) (one-way ANOVA, $P<0.05$ ) (Figure 1). In addition, the age at the time of cataract surgery showed significant differences with respect to the grading of cortical cataracts $(\mathrm{C})$ (one-way ANOVA, $P<0.05$ ) and the grading of $\mathrm{P}$ cataract (one-way ANOVA, $P<0.05$ ) (Figure 1). There were correlations among older ages at the time of cataract surgery and higher NC, C, and P LOCSIII scores (Pearson correlation coefficient; $R=0.399 ; P<0.001$; $R=0.479 ; P<0.001 ; R=0.410 ; P<0.001$, respectively).

We compared miR-34a levels between each age group and found that the older the patient, the greater the miR-34a level. The mean miR-34a level of all patients was $1.18(\mathrm{SE} \pm 0.01)$ and the mean miR-34a levels in patients older than 85 years (mean $\pm \mathrm{SE}, 1.30 \pm 0.03$ ) was significantly different from those in patients whose age was between 75 and 84 years (mean \pm SE, $1.24 \pm 0.03$ ) (ANOVA post hoc Bonferroni's test, $P<0.001$ ). In addition, the mean miR-34a levels of the patients 


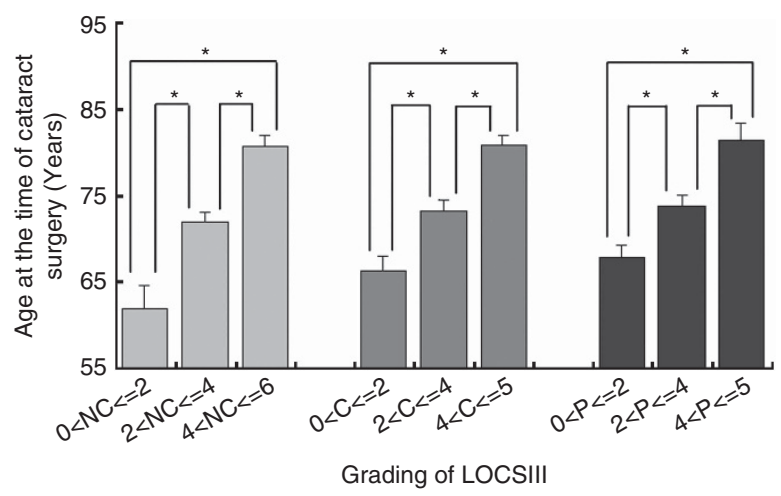

Figure 1 Differing severity of age-related cataract groups (NC, $\mathrm{C}$, and P groups obtained from the Grading of Lens Opacities Classification System version III, LOCSIII), according to subtype and age of patients at the time of cataract surgery. Significantly higher grading of age-related cataracts correlated with older age at the time of surgery $\left({ }^{*} \mathrm{P}<0.001\right)$.

between the age of 75 and 84 years were significantly different from those of the patients aged between 65 and 74 years (mean $\pm \mathrm{SE}, 1.15 \pm 0.02$ ) (ANOVA post hoc Bonferroni's test, $P<0.001)$. There was also a significant difference between the mean miR-34a levels of patients aged $65-74$ years and those of patients aged 55-64 years (mean $\pm \mathrm{SE}, 1.03 \pm 0.02$ ) (ANOVA post hoc Bonferroni's test, $P<0.001$ ) (data not shown). A scatter plot showed a moderate correlation between age at the time of cataract surgery and miR-34a levels in the lens epithelium (Pearson correlation coefficient $R=0.683 ; P<0.001$, Figure 2).

In NC cataracts, there was a moderate correlation between miR-34a levels and lens opacity severity (Pearson correlation coefficient $R=0.606 ; P<0.001$, Figure 3). Moreover, there were moderate correlations between miR-34a levels and severity of $C$ cataracts (Pearson correlation coefficient $R=0.575 ; P<0.001$ ) and between miR-34a levels and $P$ cataract severity (Pearson correlation coefficient $R=0.518 ; P<0.001$ ).

To further examine relations among levels of miR-34a expression levels, age of patients, and different levels of cataracts, we stratified patients into nine subgroups according to their levels of cataracts. It was noted that in each subgroup, miR-34a expression levels increased as older age of patients (Figure 4a). Then, in each subgroup, we compared miR-34a expression levels between samples from male and female subjects (Figure $4 \mathrm{~b}$ ). There was no significant difference between different gender samples in any subgroup of our study.

\section{Discussion}

Cataract, the first leading cause of blindness in the world, accounts for $47.8 \%$ of blindness that equals 37 millions blind people in the world. ${ }^{8}$ Notably, $90 \%$ of

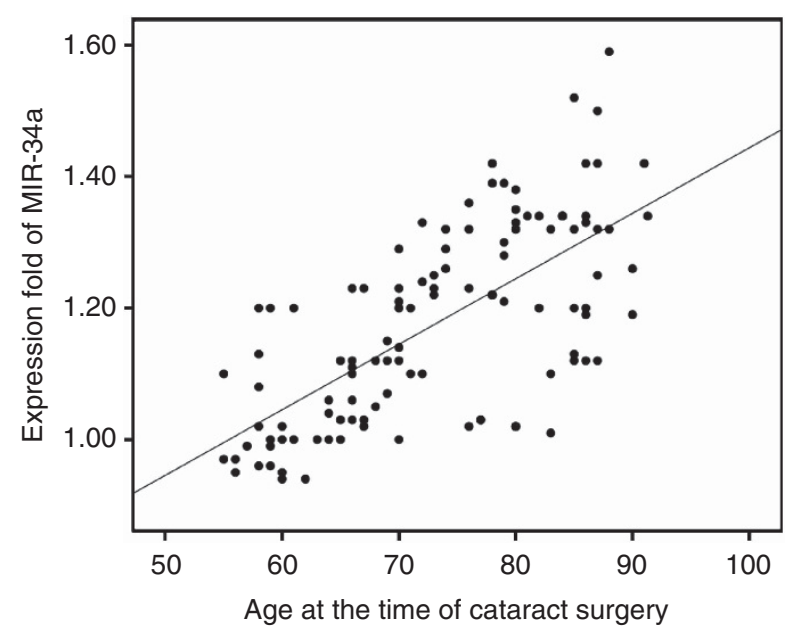

Figure 2 Relationship between age and miR-34a expression in cataract patients over 55 years of age. Each dot indicates miR-34a expression level in a single patient. Linear regression showed a significant positive correlation between miR-34a expression and age $(R=0.683, P<0.001)$. Expression ratio of miR-34a / RUN6B in control patients was defined as 1 .

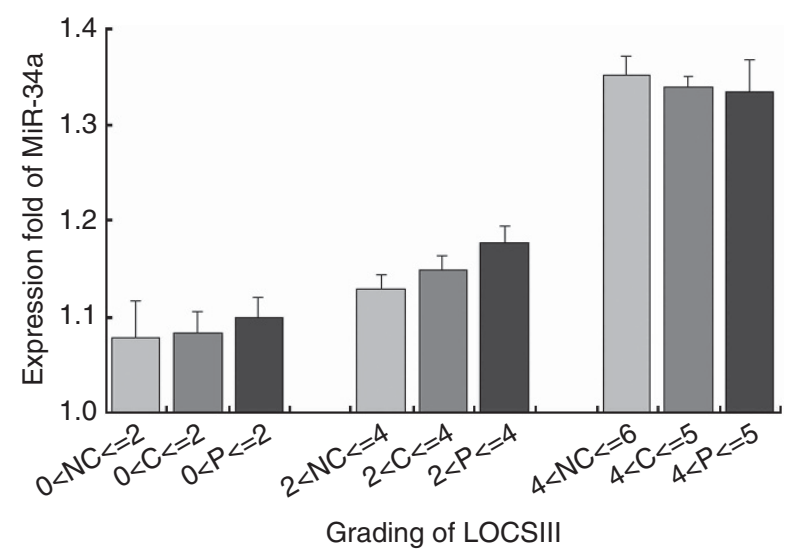

Figure 3 Relative miR-34a expression according to the severity of NC, C, and P. Real-time PCR revealed that there was a significant association between higher expression of miR-34a level and a higher severity score of $\mathrm{NC}, \mathrm{C}$, and P cataracts (Pearson correlation coefficient; $R=0.606,0.575$, and 0.515, respectively). Expression ratio of miR-34a/RUN6B in control patients was defined as 1 .

cataract-related blindness are in the developing countries. ${ }^{8}$ Most cataracts are age-related; however, there are several risk factors about cataractogenesis. ${ }^{13,14}$ Previous studies focused on prevalence differences of three specific types of lens opacity: nuclear sclerosis, C cataract, and $\mathrm{P}$ cataract. Owing to variation of subject race and age, nuclear sclerosis is most commonly noted in Canadian, Chinese, and American whites, ${ }^{15-17}$ while African, Japanese, and Singaporean present higher prevalence of $\mathrm{C}$ cataract than nuclear sclerosis. ${ }^{16}$ However, there is scant information about certain factors among different types of lens opacity. Our study enrolled 

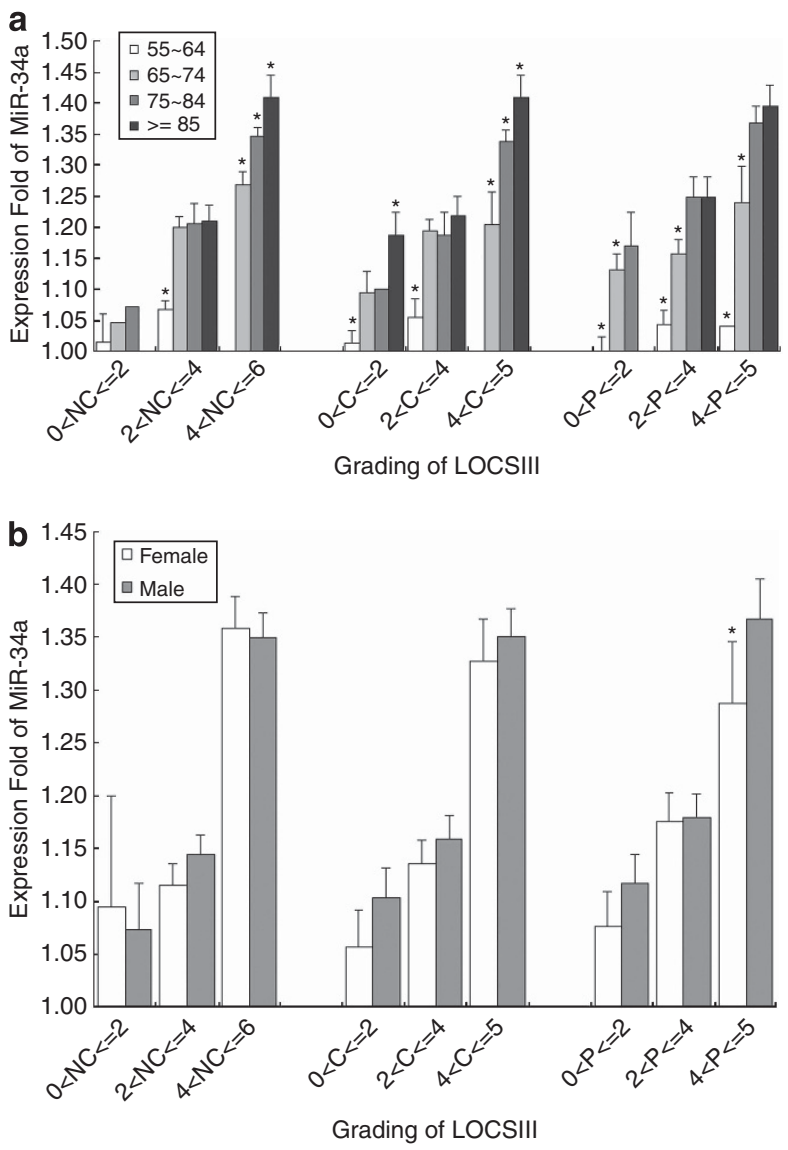

Figure 4 (a) MiR-34a expression levels in each age group stratified according to different levels of cataracts in NC, C, and P. Real-time PCR revealed that there was an association between higher expression of miR-34a level and an older age group. (b) Comparison of miR-34a expression levels between male and female subject samples stratified according to different levels of cataracts. There is no significant difference in any of subgroup. Expression ratio of miR-34a/RUN6B in control patients was defined as $1,{ }^{*} P<0.001$.

Chinese patients aged 55 and older with age-related cataract, and analyzed detailed information among individual grades of cataracts according to LOC III. We found that cataract in all subtypes of cataracts got more severe and higher miR-34a expression levels as subjects aged older. Gender is proposed as a risk factor to cataract formation; ${ }^{13}$ however, we could not find such results from our study (Figure $4 \mathrm{~b}$ ). We believed a larger scale of further study on miR-34a may be needed to answer the question.

Some confirmed risk factors to cataract formation are related to oxidative stress, such as chronic ultraviolet light exposure, smoking, and diabetes. ${ }^{14,18}$ Oxidative stress is proved to cause DNA damage and subsequent cataract formation. ${ }^{19,20}$ Blue Mountain Eye Study proved antioxidants intake, including beta-carotene, zinc, and vitamins $\mathrm{A}, \mathrm{C}$, and $\mathrm{E}$, reduced nuclear sclerosis cataract formation. ${ }^{21}$ Sorte et $a l^{20}$ examined DNA damage amounts in lens epithelial cells right after cataract surgery and found that DNA damage products were noted maximally in C cataract subject samples.

Those studies disclosed that oxidative stress prevention may be related to cataract prevention. Recently, one study on Caenorhabditis elegans with miR-34 loss-of-function mutation showed that subjects extend their lifespan, and increases resistance to oxidative stress. ${ }^{22}$ Taken together, we supposed that miR-34a may have their role in age-related catracts and from our study, we proposed that miR-34a is correlated with age of patients (Figure 2) and severity of cataracts (Figure 4a).

MiR-34a is a microRNA that regulates biological processes through complementary binding to its target at the $3^{\prime}$ UTR site. ${ }^{23}$ MiR-34a also interferes with the cell cycle and proptosis via the p53 pathway in cancer cells. ${ }^{24-26}$ MiR-34a was recently reported to be involved in senescence. Upregulation of miR-34a was observed in a premature senescence model induced by hydrogen peroxide $^{27}$ and, alternatively, antisense inhibition of miR-34a hindered the onset of replicative senescence. ${ }^{28}$ Zhao $e t ~ a l^{12}$ studied bone-marrow-derived endothelial progenitor cells (EPCs) from rats and found that overexpression of miR-34a led to the inhibition of EPC-mediated angiogenesis and induction of senescence by suppressing SirT1. In addition, Ito $e t a^{29}$ found that miR-34a increased with age in endothelial cells in senescent human umbilical cord vein endothelial cells and in the hearts and spleens of older mice. However, there is no study yet about correlation between miR-34a expression and ocular senescent tissues.

Our current study focused on miR-34a expression in the lens epithelium of age-related cataracts for investigating their role in the ocular aging process. There were significant correlations among $\mathrm{NC}, \mathrm{C}$, and $\mathrm{P}$ cataracts and age at the time of surgery (Figure 1). We also investigated the association between miR-34a expression in the lens epithelium and cataract subtypes and severity, which was classified according to LOCSIII. Our results show that the level of miR-34a expression in the lens epithelium increased with aging (Figure 2) and with the increase in the severity of NC, C, and P cataracts. (Figure 3) We believe that this is the first report describing a role for miR-34a in the senescence of aging lenses.

Age-related cataract formation involves several mechanisms, including ROS accumulation. ${ }^{9} \mathrm{UV}$ exposure and cigarette smoking are two examples of ROS-generating stress resources. Lou et al ${ }^{30}$ used hydrogen peroxide exposure to generate ROS in rat lenses and found that most of the ROS accumulated in the lens epithelium and this resulted in a cataract. Grape 
seed proanthocyanidin extract is known to protect lens epithelial cells from ROS damage ${ }^{31}$ in a dose-dependent manner and it exerts its effects via nuclear SirT1 expression. ${ }^{32}$ Recently, microRNAs, such as let-7 members, have been shown to have a role in newt lens regeneration. ${ }^{33,34}$ MiR-34a participates in the aging process by suppression of SirT1 expression. ${ }^{2,35}$ Ito et $a l^{29}$ demonstrated miR-34a regulates endothelial senescene directly through SirT1 that overexpression of miR-34a can reduce SirT1 expression and increase senescene; conversely, SirT1 expression will increase if miR-34a is knocked down. From our prior study, ${ }^{10}$ we found that decreased expression of SirT1 correlated with the severity of age-related cataracts, age at the time of surgery, and with NC, C, and P subtypes. In this study, we found that miR-34a expression positively correlated with the cataract severity according to LOCSIII classification (Figure 1). In our current study, our results support the hypothesis that miR-34a expression increases with aging ( $R=0.683$; Figure 2$)$. This result implies that miR-34a expression in the ocular aging process occurs in parallel with the aging process of the entire body through miR-34a-SirT1 pathway. We also stratified the agerelated cataracts into different subtypes according to LOCSIII and found that miR-34a positively correlated with the severity of all subtypes (Figure 3). Ito et al ${ }^{29}$ used population doubling time as a time course to follow miR-34a expression, whereas our study showed that the LOCSIII system correlated with miR-34a expression in aging human lenses.

In conclusion, in order to understand miR-34a expression specifically in human age-related cataracts, we evaluated miR-34a expression in lens epithelial cells obtained via cataract surgeries. We discovered that miR-34a expression not only correlated with age at the time of surgery but also correlated with the different severity of aging cataracts in nuclear, C, or P subtypes. Given these results, it is apparent that miR-34a expression has a role in lens senescence and may be a target for scavengers of oxidative stresses in the human ocular aging process.

\section{Summary}

What was known before

- Senile cataracts were known to be age-related and microRNA-34a recently is studied to be related to tissue senescence.

What this study adds

- This study disclosed the role of microRNA-34a in ocular aging tissue. MicroRNA-34a expression levels were shown correlations among different lens opacity severities in nuclear cataract, cortical, and posterior subcapsular cataracts.

\section{Conflict of interest}

The authors declare no conflict of interest.

\section{Acknowledgements}

This work was assisted in part by the Division of Experimental Surgery of the Department of Surgery, Taipei Veterans General Hospital. This study was supported by the National Science Council (98-2314-B341-001-MY3, 99-2314-B-075-005-MY3 and NSC 101-2314B-341 -003 -MY3), Taipei Veterans General Hospital (C1-099/ER3-001), Taipei City Hospital (96/97/98/ 099XDAA00102/10001-62-042), Shin-Kong Wu Ho-Su Memorial Hospital (SKH-8302-97-DR-25 and SKH-830298-DR-26), Zuoying Armed Forces General Hospital (9502/9696/9719), Cheng-Hsin General Hospital (CHGH-100-01, 100-19, 100-20, 100-22, and 100-40) and Yen-Tjing Ling Medical Foundation (96/97/98), Taiwan.

\section{References}

1 Zhao C, Sun G, Li S, Lang MF, Yang S, Li W et al. MicroRNA let- $7 \mathrm{~b}$ regulates neural stem cell proliferation and differentiation by targeting nuclear receptor TLX signaling. Proc Natl Acad Sci USA 2010; 107: 1876-1881.

2 Yamakuchi M, Ferlito M, Lowenstein CJ. miR-34a repression of SIRT1 regulates apoptosis. Proc Natl Acad Sci USA 2008; 105: 13421-13426.

3 Leung AK, Sharp PA. MicroRNA functions in stress responses. Mol Cell 2010; 40: 205-215.

4 Tazawa H, Tsuchiya N, Izumiya M, Nakagama H. Tumor-suppressive miR-34a induces senescence-like growth arrest through modulation of the E2F pathway in human colon cancer cells. Proc Natl Acad Sci USA 2007; 104: 15472-15477.

5 Bartel DP. MicroRNAs: genomics, biogenesis, mechanism, and function. Cell 2004; 116: 281-297.

6 Bartel DP. MicroRNAs: target recognition and regulatory functions. Cell 2009; 136: 215-233.

7 Chen LH, Chiou GY, Chen YW, Li HY, Chiou SH. MicroRNA and aging: a novel modulator in regulating the aging network. Ageing Res Rev 9(Suppl 1): S59-S66.

8 Resnikoff S, Pascolini D, Etya'ale D, Kocur I, Pararajasegaram R, Pokharel GP et al. Global data on visual impairment in the year 2002. Bull World Health Organ 2004; 82: 844-851.

9 Truscott RJ. Age-related nuclear cataract-oxidation is the key. Exp Eye Res 2005; 80: 709-725.

10 Lin TJ, Peng CH, Chiou SH, Liu JH, Lin Chung W, Tsai CY et al. Severity of lens opacity, age, and correlation of the level of silent information regulator $\mathrm{T} 1$ expression in age-related cataract. J Cataract Refract Surg 37: 1270-1274.

11 Chylack Jr LT, Wolfe JK, Singer DM, Leske MC, Bullimore MA, Bailey IL et al. The Lens Opacities Classification System III. The Longitudinal Study of Cataract Study Group. Arch Ophthalmol 1993; 111: 831-836.

12 Zhao T, Li J, Chen AF. MicroRNA-34a induces endothelial progenitor cell senescence and impedes its angiogenesis via 
suppressing silent information regulator 1. Am J Physiol Endocrinol Metab 2010; 299: E110-E116.

13 Taylor HR. Epidemiology of age-related cataract. Eye (Lond) 1999; 13(Pt 3b): 445-448.

14 West S. Epidemiology of cataract: accomplishments over 25 years and future directions. Ophthalmic Epidemiol 2007; 14: 173-178.

15 Tsai SY, Hsu WM, Cheng CY, Liu JH, Chou P. Epidemiologic study of age-related cataracts among an elderly Chinese population in Shih-Pai, Taiwan. Ophthalmology 2003; 110: 1089-1095.

16 Abraham AG, Condon NG, West Gower E. The new epidemiology of cataract. Ophthalmol Clin North Am 2006; 19: 415-425.

17 Machan CM, Hrynchak PK, Irving EL. Modeling the prevalence of age-related cataract: Waterloo Eye Study. Optom Vis Sci 2011; 89(2): 130-136.

18 Agte VV, Tarwadi KV. Combination of diabetes and cataract worsens the oxidative stress and micronutrient status in Indians. Nutrition 2008; 24: 617-624.

19 Ates O, Alp HH, Kocer I, Baykal O, Salman IA. Oxidative DNA damage in patients with cataract. Acta Ophthalmol 2010; 88: 891-895.

20 Sorte K, Sune P, Bhake A, Shivkumar VB, Gangane N, Basak A. Quantitative assessment of DNA damage directly in lens epithelial cells from senile cataract patients. Mol Vis 2011; 17: $1-6$

21 Tan AG, Mitchell P, Flood VM, Burlutsky G, Rochtchina E, Cumming RG et al. Antioxidant nutrient intake and the long-term incidence of age-related cataract: the Blue Mountains Eye Study. Am J Clin Nutr 2008; 87: 1899-1905.

22 Yang J, Chen D, He Y, Melendez A, Feng Z, Hong Q et al. MiR-34 modulates Caenorhabditis elegans lifespan via repressing the autophagy gene atg9. Age (Dordr) 2013; 35(1): 11-22.

23 Ambros V. MicroRNAs: tiny regulators with great potential. Cell 2001; 107: 823-826.

24 Christoffersen NR, Shalgi R, Frankel LB, Leucci E, Lees M, Klausen $\mathrm{M}$ et al. p53-independent upregulation of miR-34a during oncogene-induced senescence represses MYC. Cell Death Differ 17: 236-245.
25 Mraz M, Pospisilova S, Malinova K, Slapak I, Mayer J. MicroRNAs in chronic lymphocytic leukemia pathogenesis and disease subtypes. Leuk Lymphoma 2009; 50: 506-509.

26 Chen QR, Yu LR, Tsang P, Wei JS, Song YK, Cheuk A et al. Systematic proteome analysis identifies transcription factor YY1 as a direct target of miR-34a. J Proteome Res 2011; 10: 479-487.

27 Maes OC, Sarojini H, Wang E. Stepwise up-regulation of microRNA expression levels from replicating to reversible and irreversible growth arrest states in WI-38 human fibroblasts. J Cell Physiol 2009; 221: 109-119.

28 Fujita K, Mondal AM, Horikawa I, Nguyen GH, Kumamoto $\mathrm{K}$, Sohn JJ et al. p53 isoforms Delta133p53 and p53beta are endogenous regulators of replicative cellular senescence. Nat Cell Biol 2009; 11: 1135-1142.

29 Ito T, Yagi S, Yamakuchi M. MicroRNA-34a regulation of endothelial senescence. Biochem Biophys Res Commun 398: 735-740.

30 Lou MF, Xu GT, Cui XL. Further studies on the dynamic changes of glutathione and protein-thiol mixed disulfides in $\mathrm{H} 2 \mathrm{O} 2$ induced cataract in rat lenses: distributions and effect of aging. Curr Eye Res 1995; 14: 951-958.

31 Barden CA, Chandler HL, Lu P, Bomser JA, Colitz CM. Effect of grape polyphenols on oxidative stress in canine lens epithelial cells. Am J Vet Res 2008; 69: 94-100.

32 Lee YA, Cho EJ, Yokozawa T. Protective effect of persimmon (Diospyros kaki) peel proanthocyanidin against oxidative damage under $\mathrm{H} 2 \mathrm{O} 2$-induced cellular senescence. Biol Pharm Bull 2008; 31: 1265-1269.

33 Tsonis PA, Call MK, Grogg MW, Sartor MA, Taylor RR, Forge A et al. MicroRNAs and regeneration: Let-7 members as potential regulators of dedifferentiation in lens and inner ear hair cell regeneration of the adult newt. Biochem Biophys Res Commun 2007; 362: 940-945.

34 Nakamura K, Maki N, Trinh A, Trask HW, Gui J, Tomlinson $\mathrm{CR}$ et al. miRNAs in newt lens regeneration: specific control of proliferation and evidence for miRNA networking. PLoS One 5: e12058.

35 Chang TC, Wentzel EA, Kent OA, Ramachandran K, Mullendore $\mathrm{M}$, Lee $\mathrm{KH}$ et al. Transactivation of miR-34a by p53 broadly influences gene expression and promotes apoptosis. Mol Cell 2007; 26: 745-752. 